\title{
Return of the math: Markov blankets, dynamical systems theory, and the bounds of the mind
}

\author{
Lincoln J Colling \\ School of Psychology, University of Sussex \\ Commentery on Bruineberg. J., Dolega, K., Dewhurst, J., and Baltieri, M. (2021). The \\ emperor's new Markov blankets, Behavioral and Brain Sciences
}

\begin{abstract}
Bruineberg and colleagues highlight work using Markov blankets to demarcate the bounds of the mind. This echoes earlier attempts to demarcate the bounds of the mind from a dynamical systems perspective. Advocates of mechanistic explanation have challenged the dynamical approach to independently motivate the application of the formalism, a challenge that Markov blanket theorists must also meet.
\end{abstract}

The target article highlights work by, for example, Kirchhoff and Kiverstein (2019) and Ramstead et al. (2021) where attempts have been made to invoke the mathematical formalism of Markov blankets to justify claims about the bounds of the mind. The strategy highlighted in the target article is, however, not a new one. Rather, it echos earlier approaches by advocates of dynamical systems theory to similarly use mathematical models of a system's behaviour to justify claims about the bounds of the mind. By placing the target article in the context of this earlier work it might be possible to bring into sharper relief the issues raised in the target article and, more positively, sketch a way forward for Markov blanket theorists.

One well known model from the dynamical approach to cognition is the Haken-KelsoBunz model (Haken et al., 1985), which arose out of empirical work on the dynamics of interlimb coordination. For example, Kelso (1984) observed that when people rotated their wrists in an anti-phase pattern while gradually increasing the cycling speed a critical point was reached after which there was a rapid breakdown in the anti-phase pattern with coordination rapidly being reestablished in an in-phase pattern. The model was able to formalise this phenomena using the tools of dynamical modelling resulting in a model containing two parameters: one for cycling speed and one for the relative phase between the two limbs. The power of this model is its generality. Not only can it capture the dynamics of interlimb coordination but it can also be applied to phenomena like socially coordinated motor behaviour. For example, Schmidt et al. (1990) found that two people seated next to each other, and engaging in anti-phase leg swings, exhibit the same dynamics as inter-limb coordination.

E-mail: lincoln@colling.net.nz 
The Haken-Kelso-Bunz model is an example of a non-linear dynamical system. As Chemero (2011) argues, only linear systems are decomposable while non-linear systems are non-decomposable. The upshot of this non-decomposibility is that the dynamics of non-linear systems must be modelled in terms of global collective variables, and that it is not possible to model the system dynamics in terms of separate component parts. That is, the mathematical formalism enforces viewing the two people engaged in inter-personal coordination as a single unified system. The boundaries of this system are not located within a single person but encompass both people. Drawing the boundaries of the system at the edge of a person's skull amounts to splitting the system, a move prohibited by the formalism. The move here is echoed by Kirchhoff and Kiverstein (2019) and Ramstead et al. (2021) outlined by the target article. The mathematical formalism of nonlinearly coupled dynamical systems is replaced with the Markov blanket formalism, but the consequence is the same. The formalism used to model the system defines the bounds of the system.

This move is not unproblematic. The explanatory status of dynamical models has been questioned by those advocating for mechanistic explanation (Colling \& Williamson, 2014; Kaplan \& Bechtel, 2011; Kaplan \& Craver, 2011). Although it's not possible to replay these arguments here, I will pick out one point that turns on this explanatory worry. The coupling-induced synchronisation observed in intra- and inter-personal limb movements is also found in other physical systems including ostensibly non-cognitive systems like pendulum clocks. Although the model accurately predicts the dynamics of these systems, the model itself, by avoiding reference to the physical facts of the system, does not allow one to predict which systems might exhibit the relevant dynamics. However, if one does examine the physical facts of the system then it is evident why some systems exhibit the relevant dynamics and others do not. In the example of coupled pendulum clocks an explanation that makes reference to the physical facts of the system, their parts, and their interactionsthat is, a mechanistic explanation - provides reasons why certain arrangements of pendulum clocks exhibit the relevant dynamics while other arrangements do not. For example, a sketch of a mechanistic explanation might reference vibrations produced by the clocks and the role the wall plays in transmitting vibrations between clocks. This in turn provides an explanation for why clocks placed side-by-side on the same wall exhibit the relevant dynamics while clocks placed on opposite walls do not. Mechanistic explanations might similarly be furnished for why particular limb movements or interpersonal actions exhibit the relevant dynamics. The fact that the system exhibits these dynamics is only part of the story. What is missing is an explanation of why the system should exhibit these dynamics in the first place. The upshot of this is that what licenses application of the model (and what licenses demarcating the boundaries of the system) are some set of facts about the mechanism.

The move by Kirchhoff and Kiverstein (2019) and Ramstead et al. (2021) to demarcate the boundaries of the mind gives rise to a similar worry. Is there some set of explanatory facts that licences placing the Markov blanket at the brain, the skin, or at any other "boundary"? On this, the answer is not clear. For example, Kirchhoff and Kiverstein (2019) reject the idea of explanation dependent boundaries while Ramstead et al. (2021) appears to at least partially endorse the idea. Kirchhoff et al. (2018), go further and explicitly reference a mechanism sketch in deciding on the location of the Markov blanket (using the example of coupled pendulums). But as the example from dynamical systems theory shows, the formalism itself does not license predictions about which systems are 
amenable to the formalism and which are. Rather, these predictions are made independently of the formalism on, for example, mechanistic grounds. The Markov blanket theorist is presented with the same challenge. That is, to provide an explanation or prediction of which systems are amenable to the formalism - or because the formalism is applicable to every "thing" (Friston, 2019), which systems are amenable to specific applications of the formalism independent of the particular application of the formalism itself.

\section{References}

Chemero, A. (2011). Radical embodied cognitive science. MIT press.

Colling, L. J., \& Williamson, K. (2014). Entrainment and motor emulation approaches to joint action: Alternatives or complementary approaches? Frontiers in Human Neuroscience, 8, 754. https://doi.org/10.3389/fnhum.2014.00754

Friston, K. (2019). A free energy principle for a particular physics. arXiv: 1906.10184 [q-bio].

Haken, H., Kelso, J. A. S., \& Bunz, H. (1985). A theoretical model of phase transitions in human hand movements. Biological Cybernetics, 51(5), 347-356. https://doi.org/ $10.1007 / \mathrm{BF} 00336922$

Kaplan, D. M., \& Bechtel, W. (2011). Dynamical Models: An Alternative or Complement to Mechanistic Explanations? Topics in Cognitive Science, 3(2), 438-444. https: //doi.org/10.1111/j.1756-8765.2011.01147.x

Kaplan, D. M., \& Craver, C. F. (2011). The Explanatory Force of Dynamical and Mathematical Models in Neuroscience: A Mechanistic Perspective. Philosophy of Science, 78(4), 601-627. https://doi.org/10.1086/661755

Kelso, J. A. (1984). Phase transitions and critical behavior in human bimanual coordination. The American Journal of Physiology, 246, R1000-1004. https://doi.org/10.1152/ ajpregu.1984.246.6.R1000

Kirchhoff, M. D., \& Kiverstein, J. (2019). How to determine the boundaries of the mind: A Markov blanket proposal. Synthese, 1-20.

Kirchhoff, M. D., Parr, T., Palacios, E., Friston, K., \& Kiverstein, J. (2018). The Markov blankets of life: Autonomy, active inference and the free energy principle. Journal of The Royal Society Interface, 15(138), 20170792. https://doi.org/10.1098/rsif.2017. 0792

Ramstead, M. J. D., Kirchhoff, M. D., Constant, A., \& Friston, K. J. (2021). Multiscale integration: Beyond internalism and externalism. Synthese, 198(1), 41-70. https: //doi.org/10.1007/s11229-019-02115-x

Schmidt, R. C., Carello, C., \& Turvey, M. T. (1990). Phase transitions and critical fluctuations in the visual coordination of rhythmic movements between people. Journal of Experimental Psychology: Human Perception and Performance, 16(2), 227-247. https://doi.org/10.1037/0096-1523.16.2.227 